\title{
p53 mutation, deprivation and poor prognosis in primary breast
}

\section{cancer}

\section{Baker,, , PR Quinlan', N Patten², A Ashfield', L-J Birse-Stewart-Bell', C McCowan ${ }^{3}$, J-C Bourdon', CA Purdie ${ }^{4}$, LB Jordan ${ }^{4}$, JA Dewar ${ }^{5}, L$ Wu$^{2}$ and AM Thompson'}

'Department of Surgery and Molecular Oncology, Dundee University, Ninewells Hospital and Medical School, Dundee DDI 9SY, UK; ${ }^{2}$ Roche Molecular Systems, 4300 Hacienda Drive, Pleasanton, CA 94588, USA; ${ }^{3}$ Health Informatics Centre, Dundee University, Mackenzie Building, Ninewells Hospital and Medical School, Dundee DD2 4BF, UK; ${ }^{4}$ Department of Pathology, Ninewells Hospital and Medical School, Dundee DDI 9SY, UK; ${ }^{5}$ Department of Oncology, Ninewells Hospital and Medical School, Dundee DDI 9SY, UK

BACKGROUND: The deprivation gap for breast cancer survival remains unexplained by stage at presentation, treatment, or comorbidities. We hypothesised that p53 mutation might contribute to the impaired outcome observed in patients from deprived communities.

METHODS: p53 mutation status was determined using the Roche Amplichip research test in 246 women with primary breast cancer attending a single cancer centre and related to deprivation, pathology, overall, and disease-free survival.

RESULTS: 553 mutation, identified in 64/246 (26\%) of cancers, was most common in 10 out of 17 (58.8\%) of the lowest (I0th) deprivation decile. Those patients with $p 53$ mutation in the I0th decile had a significantly worse disease-free survival of only $20 \%$ at 5 years (Kaplan-Meier logrank $\chi^{2}=6.050, P=0.014$ ) and worse overall survival of $24 \%$ at 5 years (Kaplan-Meier logrank $\chi^{2}=6.791$, $P=0.009$ ) than women of deciles I - 9 with $p 53$ mutation (c.f. $56 \%$ and $72 \%$, respectively) or patients in the I0th decile with wildtype $p 53$ (no disease relapse or deaths).

CONCLUSION: $p 53$ mutation in breast cancer is associated with socio-economic deprivation and may provide a molecular basis, with therapeutic implications, for the poorer outcome in women from deprived communities.

British Journal of Cancer (2010) 1 02, 719-726. doi:10.1038/sj.bjc.6605540 www.bjcancer.com

Published online 26 January 2010

(c) 2010 Cancer Research UK

Keywords: p53; primary breast cancer; socio-economic deprivation; overall survival; disease-free survival

Deprivation has been linked to a wide range of disease (Banks et al, 2006) and to poor outcome for patients diagnosed with cancer, including breast cancer (Schrijvers et al, 1995; Twelves et al, 2001; Thomson et al, 2004; Mullee et al, 2004), colorectal cancer (McMillan et al, 2003), and melanoma (MacKie and Hole, 1996).

Although the incidence of breast cancer has risen more rapidly in women with a high socio-economic status than those from deprived areas, the difference in survival - the 'deprivation gap' has not changed (Quinn et al, 2008) and cannot be attributed to the higher uptake of breast screening in higher socio-economic groups (Thomson et al, 2004). Possible explanations include:

(a) later presentation (Kogevinas and Porta, 1997), although not substantiated in all populations (Sant et al, 1998);

(b) under-treatment, evident in Scotland in the 1980s (Twelves et al, 2001) but not pertinent by 1994 (Thomson et al, 2004);

(c) reproductive factors, such as age at first pregnancy, menopause, and use of hormone replacement therapy, which even where highlighted would not account for all the difference (Thomson et al, 2001);

*Correspondence: Dr L Baker; E-mail: I.baker@dundee.ac.uk Revised 25 November 2009; accepted 16 December 2009; published online 26 January 2010 (d) biological differences between breast cancer arising in women according to socio-economic group. For example, a higher incidence of oestrogen receptor (ER)-positive tumours in higher social groups in some (Thomson et al, 2001) but not all series (Al Murri et al, 2004).

Given the uncertainty for the underlying basis for the deprivation gap, we considered the $p 53$ gene a candidate molecular marker that might account for the biological differences.

Briefly, p53 has pleiotropic functions including responses to cellular stresses leading to cell cycle arrest, apoptosis or cellular senescence (Staples et al, 2008). p53 also fulfills functions during development in normal tissues (Vousden and Lane, 2007) and in response to inflammation (Staib et al, 2005). The role of p53 in breast cancer has been the subject of much debate (Staples et al, 2008) with recent clarity that $p 53$ mutation is a marker of biologically aggressive disease (Olivier et al, 2006; Staples et al, 2008) and response to therapy (Staples et al, 2008).

Given the uncertainties around the reasons for the poorer outcome for women with breast cancer from deprived areas and the continuing interest around the roles of $p 53$ in breast cancer, we set out to test the hypothesis that breast cancer in deprived women is biologically more aggressive than breast cancer in less deprived women and that $p 53$ mutation may account, at least in part, for this association. 


\section{MATERIALS AND METHODS}

\section{Patients and tissues}

A total of 246 Caucasian patients undergoing resection for primary, previously untreated, operable breast cancer tissue between 1997 and 2001, with frozen tissue stored in the regional tissue bank and a minimum follow-up period of at least 5 years (or to death), but otherwise unselected, were studied. All women, regardless of social category, were diagnosed and treated at the regional cancer centre served by a single multi-disciplinary team. The total sample size of 246 was estimated on the basis of deprivation deciles of minimum size 15 patients. Local research ethics tissue bank committee approval of the project was obtained.

Clinical and pathological data were collected prospectively and included pathological tumour size, histological grade, ER, progesterone receptor and HER2 (Human Epidermal growth factor Receptor 2) receptor status, pathological lymph node status, adjuvant therapies, recurrence(s) and survival.

Breast tissue was macro-dissected by a specialist breast pathologist immediately after operation and tumour was snapfrozen in liquid nitrogen before storage at $-80^{\circ} \mathrm{C}$. Total genomic DNA was isolated using MagAttract DNA Mini M48 Kit on a BioRobotM48 (Qiagen, Crawley, West Sussex, UK) according to the manufacturer's protocol. The quantity and purity of DNA was determined using a NanoDrop ND-1000 spectrophotometer (Labtech International, Ringmer, East Sussex, UK).

\section{AmpliChip p53 Test}

p53 mutation status was determined using the Roche p53 Amplichip research test (Roche Molecular Systems, Pleasanton, CA, USA) from $100 \mathrm{ng}$ of purified genomic DNA extracted from homogenised fresh frozen tumour tissues. The AmpliChip p53 microarray consists of over 33000 probe sets of more than 220000 individual oligonucleotides tiled for a total of 1268 nucleotide positions of coding regions of exons $2-11$. A single probe set for an interrogating base position includes five probes: one probe to hybridise to the WT, three probes to detect three possible single base-pair mutations, and one probe to detect single deletion. There are at least 24 probe sets for each nucleotide position, including both sense and anti-sense probe sequences. The p53 mutation status was determined by a p53 Mutation Detection Algorithm developed for research use by Roche Molecular Systems, which is designed to detect single base-pair substitutions and single basepair deletions of a sample in a background of WT p53 DNA probe intensities.

\section{Deprivation data}

Deprivation was calculated using the Carstairs index of socioeconomic status (Carstairs and Morris, 1991) for patients who were diagnosed with breast cancer between 1997 and 2001. The Carstairs index produces deprivation scores for postcode sectors using the standard UK postcode minus the last two characters, so large areas are grouped together under a single deprivation score. The Information Services Division, Scotland (Bishop et al, 2004) has subdivided each of these areas to produce a decile score for each full postcode in Scotland ranging from 1 (most affluent) to 10 (most deprived). This decile score allows the precise linkage of patients to the correct deprivation score, and was adopted for this study.

\section{Data analyses}

Initial analyses indicated that the deprivation extremes were more likely to yield statistically significant data; indeed, deprivation categories 2-9 rarely produced statistically differing results. Moreover, deprivation category $1 \mathrm{did}$ not produce significant results compared with categories 2-9, and the deprivation results were, therefore, subsequently grouped as 1-9 and 10, unless otherwise stated.

Data were analysed for three cohorts of patients: (1) all patients in the study, (2) all patients with a $p 53$ mutation $(p 53 \mathrm{~m})$, and (3) all patients that did not have a $p 53$ mutation (WT; $p 53 \mathrm{WT}$ ).

Additional analyses were performed according to histological tumour grade (Bloom and Richardson, 1957) (graded by a specialist consultant breast pathologist); pathological tumour size (pT1, tumours $<2 \mathrm{~cm} v s$ pT2 and pT3 cancers - tumours $\geqslant 2 \mathrm{~cm}$ ); ER status (as ER negative: $0-3$ vs ER positive: $4-18$ by the Quickscore method (Detre et al, 1995)); HER2 positive (HER2 $2+$ and gene amplified on fluorescence in situ hybridisation or HER2 $3+$ on immunohistochemistry); and triple negative (as ER, progesterone receptor and HER2 negative) or not triple negative.

Treatments were analysed to assess whether survival and recurrence intervals were influenced by differences in treatment. Recurrence was defined as clinical, radiological or pathological evidence of disease recurrence. Survival was calculated from the date of surgery to the date of death (last recorded hospital visit for censored data). All deaths that were not attributable to breast cancer were censored at the date of death. Accordingly, the primary end points were breast cancer-specific overall survival and breast cancer-specific disease-free survival (respectively abbreviated to OS and DFS throughout).

Tests for statistical significance between ordinal variables were performed by two-sided Fisher's exact tests (2FET) and nonparametric Kaplan - Meier survival analyses (KM) using Mosaic, an internally developed statistical analysis system (implemented in Matlab, Mathworks, Natick, MA, USA; Version 6.5 Release 13). For all analyses, the null hypothesis was rejected at an $\alpha$-level of $10 \%$ $(P>0.10)$, observations were considered to be marginal (i.e. worthy of further analysis) for an $\alpha$-level between 5 and $10 \%$ $(0.05 \leqslant P<0.10)$, and significant at a $5 \% \alpha$-level $(P<0.05)$. Further analyses were conducted using binary logistic regression (BLR) with associated odds ratios (ORs) using Minitab (Minitab State College, PA, USA, Release 14.1) and Cox's proportional hazards regression model with associated risk ratios (RRs).

\section{RESULTS}

Deprivation category, clinical and pathological data, and p53 mutation status were successfully ascertained in all 246 patients (Table 1). The observed frequency of most variables was close to the expected in each decile (as calculated by their $\chi^{2}$ scores), and there was no gradation across deprivation deciles for any variable. However, several variables in deprivation category 10 registered significantly high or low values.

Overall, 109 of the 234 patients with tumour grade assessment were grade $3(46.6 \%)$ with significantly more (12 out of $17 ; 70.6 \%)$ grade 3 tumours in the lowest deprivation decile $(P=0.046,2 \mathrm{FET}$; $\mathrm{OR}=3.0(1.0,8.7)$; power $=0.54)$. For HER2 status, 33 out of 230 tested were HER2 positive (14.3\%), with more HER2-positive patients in deprivation category $10(6 / 16 ; 37.5 \%)$ than expected $(P=0.015,2$ FET; OR $=4.2(1.4,12.4)$; power $=0.72)$. HER2 positivity was also significantly associated with $p 53$ mutation $(P=0.003$, 2FET; OR $=3.3(1.5,7.0)$; power $=0.85)$. Age, tumour size, nodal status, ER, progesterone receptor and triple-negative cancers were not significantly associated with deprivation category.

\section{p53 mutation}

p53 mutation was detected in 64 cancers from 246 patients $(26.0 \%)$, excluding the $p 53$ polymorphism at amino-acid 72 as a neutral change in terms of p53 function. p53 mutation was 
Table I Study characteristics of the breast cancer clinical and pathological variables grouped by deprivation I -9 and 10 and by p53 mutation status

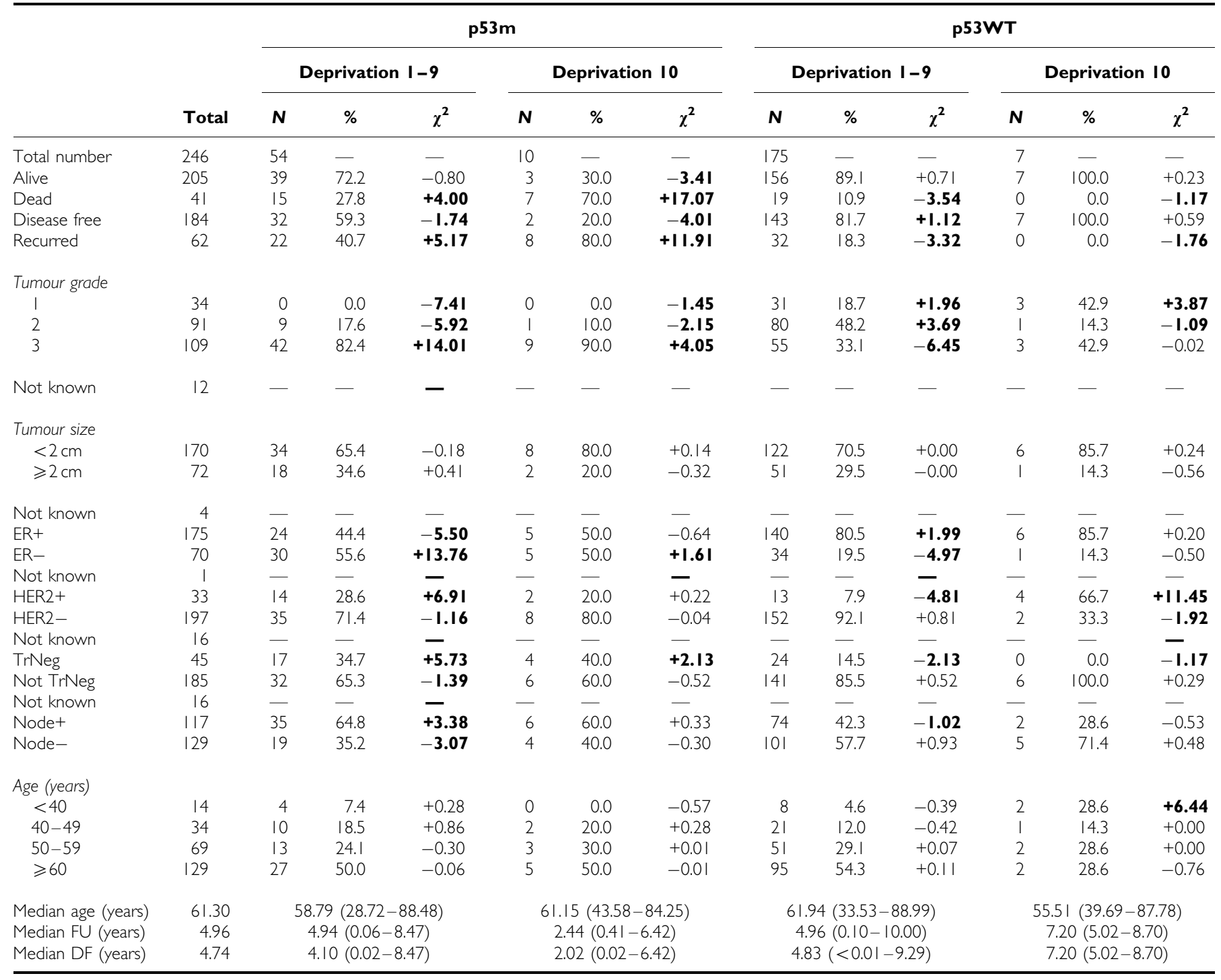

Abbreviations: $\mathrm{DF}=$ disease-free survival; $E R=$ oestrogen receptor; $F U=$ follow-up. Percentages may not sum to 100 due to rounding. The $\chi^{2}$ score, calculated across all four p53/deprivation categories, indicates the deviation of the observed from the expected frequency, in which a high score signifies a large deviation and a $\chi^{2}$ score greater than unity is deemed 'of interest', and highlighted in bold type. The $\chi^{2}$ score is marked with a ' + ' if the observed frequency exceeds the expected, and with a '-' otherwise. Median ages and follow-up were not significantly different for any of the patient groupings ( $p \geqslant 0.342$ in all cases; two-sample $t$-test and Mann-Whitney U-test).

significantly associated with a decrease in OS $(34 \%$ vs $10 \%$ dead $P<0.0001,2 \mathrm{FET} ; \mathrm{OR}=0.2(0.1,0.5)$; power $=0.98)$ and DFS $(47 \%$ vs $18 \%$ relapsed $-P<0.0001,2 \mathrm{FET} ; \mathrm{OR}=0.2(0.1,0.5)$; power $=0.99)$. Tumour $p 53$ mutation was significantly associated with grade 3 cancer $(P<0.0001, \quad 2 \mathrm{FET}$; $\mathrm{OR}=10.3(4.9,21.8)$; power $=1.00)$; axillary lymph node metastasis $(P=0.004,2 \mathrm{FET}$; $\mathrm{OR}=2.4(1.3,4.3)$; power $=0.84)$; ER-negative tumours $(P<0.0001$, 2FET; $\quad$ OR $=4.9(2.6,9.0)$; $\quad$ power $=1.00)$; HER2 expression $(P=0.003, \quad 2 \mathrm{FET} ; \quad \mathrm{OR}=3.3(1.5,7.0) ; \quad$ power $=0.85)$ and triplenegative tumours $(P=0.001, \quad$ 2FET; $\quad \mathrm{OR}=3.3(1.7,6.5)$; power $=0.92$ ). There was no association between $p 53$ mutation status and pathological tumour size or patient age.

\section{Univariate analyses}

Both OS in deprivation category $10(P=0.012,2 \mathrm{FET}$; OR $=$ $0.2(0.1,0.7)$; power $=0.74)$; and DFS $(P=0.042,2 \mathrm{FET}$; OR $=0.3$ $(0.1,0.9)$; power $=0.57)$ were worse than expected. Patients in deciles 1-9, but not decile 10, were significantly more likely to have a relapse and die if they had a grade 3 tumour, large cancer, ER-negative, HER2-positive or triple-negative cancer (KM: logrank $\chi^{2} \geqslant 5.650, P \leqslant 0.017$ in each case, Table 2). Patients with axillary metastases were more likely to have a relapse or die from breast cancer irrespective of deprivation category, with the notable exception that patients in deprivation category 10 were significantly more likely to have a relapse, but were not significantly more likely to die $\left(\chi^{2} \geqslant 4.620, P \leqslant 0.032\right.$ in each case - excepted $\chi^{2}=3.080, P=0.079$, Table 2 ). There was no association between any of the treatment regimes and deprivation.

Tumour $p 53$ mutation was more common in deprivation decile 10 (10 out of 17 patients, 58.8\%) than WT $p 53(P=0.003,2 \mathrm{FET}$; $\mathrm{OR}=4.6(1.7,12.7)$; power $=0.87)$. In the patients with $p 53$ mutation, $27.8 \%$ (15 out of 54 ) of the patients in deprivation group 1-9 died, whereas $70.0 \%$ ( 7 out of 10 ) of those in decile 10 died $(P=0.025, \quad 2 \mathrm{FET} ; \quad$ OR $=6.1(1.4,26.6) ;$ power $=0.74)$. Similarly, among p53 mutant cancers $40.7 \%$ (22 out of 54 ) of patients in 
Table 2 Results of Kaplan-Meier overall survival and disease-free survival analyses for all patients, patients in the p53 mutant and in the p53 wild-type cohorts

\begin{tabular}{|c|c|c|c|c|c|c|c|c|c|c|c|c|c|}
\hline & $\begin{array}{c}\text { Dep. } \\
\text { group }\end{array}$ & \multicolumn{6}{|c|}{ Survival } & \multicolumn{6}{|c|}{ Disease free } \\
\hline \multirow[t]{2}{*}{ p53 mutation (vs WT) } & All & 21.27 & $<0.0001$ & - & - & - & - & 23.31 & $<\mathbf{0 . 0 0 0 I}$ & - & - & - & - \\
\hline & $1-9$ & 9.96 & 0.002 & - & - & - & - & 12.10 & 0.001 & - & - & - & - \\
\hline & $1-9$ & |8.8I & $<0.0001$ & $3.55^{a}$ & $0.059^{a}$ & 9.58 & 0.002 & 13.96 & 0.0002 & $3.94^{\mathrm{a}}$ & $0.047^{a}$ & 2.97 & 0.085 \\
\hline & 10 & 1.51 & 0.219 & $0.01^{\mathrm{a}}$ & $0.937^{\mathrm{a}}$ & $N / A^{b}$ & $N / A^{b}$ & 1.31 & 0.252 & $8.20^{a}$ & $0.004^{a}$ & $N / A^{b}$ & $N / A^{b}$ \\
\hline \multirow[t]{3}{*}{ Tumour size $(<2 \mathrm{~cm} v s \geqslant 2 \mathrm{~cm})$} & All & 10.13 & 0.001 & 0.32 & 0.574 & 14.95 & 0.0001 & 8.06 & 0.005 & 2.20 & 0.138 & 5.11 & 0.024 \\
\hline & $1-9$ & II.0I & 0.001 & 0.28 & 0.596 & 14.32 & 0.0002 & 8.25 & 0.004 & 2.30 & 0.130 & 4.75 & 0.029 \\
\hline & 10 & 1.08 & 0.298 & 1.07 & 0.300 & $N / A^{b}$ & $N / A^{b}$ & 2.05 & 0.152 & 9.54 & 0.002 & $N / A^{b}$ & $N / A^{b}$ \\
\hline \multirow[t]{2}{*}{$\mathrm{ER}(+\mathrm{vs}-)$} & All & 31.65 & $<0.0001$ & 5.75 & 0.017 & 13.79 & 0.0002 & 25.06 & $<\mathbf{0 . 0 0 0 1}$ & 5.38 & 0.020 & 6.54 & 0.011 \\
\hline & $1-9$ & 27.83 & $<\mathbf{0 . 0 0 0 1}$ & 5.81 & 0.016 & 13.66 & 0.0002 & $22.7 I$ & $<0.0001$ & 6.80 & 0.009 & 6.47 & 0.011 \\
\hline \multirow{2}{*}{ Triple negative (yes vs no) } & $1-9$ & $|3.4|$ & 0.0003 & 4.92 & 0.027 & 3.39 & 0.066 & 10.29 & 0.001 & 4.44 & 0.035 & 2.15 & 0.142 \\
\hline & 10 & 2.48 & 0.115 & 0.08 & 0.774 & $\mathrm{~N} / \mathrm{A}^{\mathrm{b}, \mathrm{c}}$ & $N / A^{b, c}$ & 1.32 & 0.250 & 0.03 & 0.854 & $N / A^{b, c}$ & $\mathrm{~N} / \mathrm{A}^{\mathrm{b}, \mathrm{c}}$ \\
\hline \multirow[t]{3}{*}{ Nodal status (+ vs - ) } & All & 19.14 & $<\mathbf{0 . 0 0 0 1}$ & 5.33 & 0.021 & 9.55 & 0.002 & 15.92 & $<\mathbf{0 . 0 0 0 1}$ & 11.90 & 0.0006 & 2.07 & 0.150 \\
\hline & $1-9$ & 16.96 & $<0.0001$ & 4.72 & 0.030 & 9.21 & 0.002 & 12.42 & 0.0004 & 10.46 & 0.001 & 1.87 & 0.171 \\
\hline & 10 & 3.08 & 0.079 & 1.35 & 0.244 & $N / A^{b}$ & $N / A^{b}$ & 4.62 & 0.032 & 2.39 & 0.122 & $N / A^{b}$ & $N / A^{b}$ \\
\hline
\end{tabular}

Abbreviations: $E R=$ oestrogen receptor; $\mathrm{WT}=$ wild type. Results are also detailed by deprivation groups I - 9 and $10 .{ }^{\mathrm{a}}$ There were no $p 53$ mutation patients, who had Grade I tumours. ${ }^{b}$ All patients in deprivation group 10 without a p53 mutation survived and remained disease free. ${ }^{c}$ All patients in deprivation group 10 without a p53 mutation were free of triple-negative tumours. All analyses have one degree of freedom, and statistically significant results are highlighted in bold type.

deprivation groups 1-9 relapsed compared with $80 \%$ (8 out of 10) of those in the worst deprivation category $(P=0.025$, 2FET; $\mathrm{OR}=6.1(1.4,26.6)$; power $=0.74$; and $P=0.036$, 2FET; $\mathrm{OR}=5.8(1.1,30.0)$; power $=0.65$; respectively). However, in the p53 WT cohort, there was no such association between deprivation and OS or DFS; indeed, all seven patients with p53 WT cancers in deprivation category 10 survived and remained disease free at a median follow-up of 7.2 years (95\% CI: 5.5-8.1 years).

\section{Multivariate analyses}

The analyses of BLRs and Cox's logistic regressions (Table 3) were also performed. Death and recurrence were chosen as the response variables so that most of the $O R$ and $R R$ values (BLR and Cox's proportional hazards regression, respectively) would be greater than unity (OS and DFS OR and RR values and 95\% CI values may be obtained by inversion). Results of BLRs and Cox's proportional hazards regressions were in agreement with all analyses, and as such, the results of BLR analyses have been excluded from Table 3.

In the first two sets of analyses (top half of Table 3), deprivation groups $1-9$ and 10 were input as binary variables, as were $p 53 \mathrm{~m} /$ WT for the prediction of death and recurrence (left and right of Table 3, respectively). In the second set of analyses (bottom half of Table 3), the influence on death and recurrence of deprivation category 10 patients who had p53-mutated cancer was explored. Deprivation category 10 was a strong predictor of death and recurrence, as were large tumours, ER negativity and nodal status $(P \leqslant 0.041$ in each case). Interestingly, $p 53$ mutation was strongest as a predictor of disease recurrence, but less strong as a predictor for death.

When patients were grouped according to deprivation category 10 with a $p 53$ mutation ( $v s$ all other patients), the ability to predict death or recurrence became very strong and highly significant $(\beta \geqslant \pm 2.18$,
$P \leqslant 0.0001$ in all cases). The $\mathrm{OR}$ and $\mathrm{RR}$ values for death and recurrence were large $(\mathrm{OR}=33.4(5.2,215.0)$ and $\mathrm{OR}=19.6(3.4,114.2)$ and $\mathrm{RR}=12.4(4.8,32.3)$ and $\mathrm{RR}=8.8(3.6,21.7)$, respectively). Tumour size, ER status and nodal status all retained large $\beta$ coefficients and remained significant predictors of death and recurrence $(\beta \geqslant \pm 0.90, P \leqslant 0.026$ in all cases).

In addition, although there was no association between any of the treatment regimes and deprivation in univariate analyses, all multivariate analyses were re-run with various treatment regimes included as binary variables (data not shown). Treatment regimes were not significant, and were therefore not associated with the outcome variables.

\section{Kaplan - Meier survival analyses}

Patients from the worst deprivation decile were significantly more likely to have a relapse or die than those in deprivation groups 1-9 (5-year OS: $57 \%$ vs $86 \%$; KM: logrank $\chi^{2}=7.827, P=0.005$ for OS and KM: logrank $\chi^{2}=4.450, P=0.035$ for DFS). Similarly, patients with a mutant $p 53$ were more likely to relapse or die compared with those with WT $p 53$ (5-year OS: 65\% vs 90\%; KM: logrank $\chi^{2}=21.267, P<0.0001$ for OS and KM: logrank $\chi^{2}=23.308$, $P<0.0001$ for DFS).

Patients were divided into four groupings - each of deprivation groups 1-9 and 10 with each of $p 53$ mutation and WT - and plotted as KM analyses in Figure 1 (OS and DFS, top and bottom, respectively).

Within the decile groups (1-9 and 10), OS and DFS were both as expected - better in the p53 WT patients compared with the mutant (decile 1-9: 5 -year OS: $89 \%$ vs $72 \%$; decile 10: 5 -year OS: $100 \%$ vs $24 \%$; decile 1-9 KM: logrank $\chi^{2}=9.958, P=0.002$ and KM: logrank $\chi^{2}=12.098, P=0.0005$ for OS and DFS, respectively; decile $10 \mathrm{KM}$ : logrank $\chi^{2}=8.011, P=0.005$ and KM: logrank $\chi^{2}=9.177, P=0.002$, OS and DFS, respectively). 
Table 3 Results of Cox proportional hazards regression model, with death and recurrence as the response variables

\begin{tabular}{|c|c|c|c|c|c|c|}
\hline & \multicolumn{3}{|c|}{ Death } & \multicolumn{3}{|c|}{ Recurrence } \\
\hline Deprivation 10 (vs I-9) & 1.81 & 0.0003 & $6.1(2.3-16.2)$ & 1.31 & 0.003 & $3.7(1.6-8.9)$ \\
\hline $\mathrm{ER}(+v s-)$ & -2.50 & 0.041 & $0.1(<0.1-0.9)$ & -1.28 & 0.056 & $0.3(0.1-1.0)$ \\
\hline p53 mutation (vs WT) & 0.67 & 0.114 & $1.9(0.9-4.5)$ & 0.95 & 0.009 & $2.6(1.3-5.2)$ \\
\hline Tumour grade (3 vs I-2) & 0.90 & 0.087 & $2.5(0.9-6.9)$ & 0.18 & 0.677 & $1.2(0.5-2.7)$ \\
\hline Triple negative ( $n$ vs y) & -1.54 & 0.229 & $0.2(<0.1-2.6)$ & -0.74 & 0.325 & $0.5(0.1-2.1)$ \\
\hline Dep $10 \&$ p53 m ( $v$ rest $)$ & 2.52 & $<0.0001$ & $12.4(4.8-32.3)$ & 2.18 & $<0.0001$ & $8.8(3.6-21.7)$ \\
\hline Tumour size (large vs small) & 1.26 & 0.002 & $3.5(1.6-7.7)$ & 0.90 & 0.005 & $2.5(1.3-4.7)$ \\
\hline $\mathrm{ER}(+v s-)$ & -2.65 & 0.021 & $0.1(<0.1-0.7)$ & -1.47 & 0.023 & $0.2(0.1-0.8)$ \\
\hline Tumour grade ( 3 vs I -2 ) & 1.07 & 0.033 & $2.9(1.1-7.8)$ & 0.35 & 0.369 & $1.4(0.7-3.0)$ \\
\hline Nodal status (+ vs - ) & 1.49 & 0.002 & $4.4(1.8-\mid 1.2)$ & 1.05 & 0.002 & $2.9(1.5-5.6)$ \\
\hline
\end{tabular}

Abbreviations: $\mathrm{ER}=$ oestrogen receptor; $\mathrm{RR}=$ risk ratio; $\mathrm{WT}=$ wild type. The upper models have deprivation and $\mathrm{p} 53$ mutation status as individual binary input variables, whereas the lower models group deprivation category 10 patients that also have a $p 53$ mutation (versus all other patients) as a binary input variable. The G-statistic $\chi^{2} \geqslant 51.0$ and $P<0.000$ I in each model, with the upper models having eight degrees of freedom and the lower models having seven. A strong predictor of the response variable (death or recurrence; left and right half of the table, respectively) is one that has a large $\beta$-coefficient, whether positive or negative, and a high degree of confidence in the coefficient, $P \leqslant 0.05$. Such strong predictors are also backed by RR values with associated $95 \%$ confidence intervals that exclude unity. Indicators of strong predictors are highlighted in bold type.

Within the p53-mutant patients, those in deprivation decile 10 were more likely to have a relapse or die compared with those in deciles 1-9 (p53m: 5-year OS: $24 \%$ vs 72\%; p53m 5-year DFS: $20 \%$ vs 55\%; KM: logrank $\chi^{2}=6.791, P=0.009$ and KM: logrank $\chi^{2}=6.050, P=0.014$ for OS and DFS, respectively). In contrast, within the WT $p 53$ patients, there was no statistically significant difference between decile group $1-9$ and 10 for either OS or DFS (KM: logrank $\chi^{2}=0.917, P=0.338$ and KM: logrank $\chi^{2}=1.626$, $P=0.202$ for OS and DFS, respectively).

\section{DISCUSSION}

This study demonstrates the new finding that a worse survival and shorter disease free interval in breast cancer for the most socioeconomically deprived patients (deprivation decile 10) is associated with tumour $p 53$ mutation. This suggests the intriguing possibility of a causal link between the molecular basis of breast cancer exemplified by $p 53$ mutations and extreme deprivation, and that the biological features of breast cancer may contribute substantially to the perceived deprivation gap in survival compared with stage of presentation, co-morbidities or treatment differences (Kelsey et al, 1981; Schrijvers et al, 1997; Twelves et al, 2001; Taylor and Cheng, 2003; Thomson et al, 2004; Mullee et al, 2004).

p53 mutation in this series of patients was associated, as anticipated, with high tumour grade, axillary node metastasis, ER-negative cancers and HER2-positive cancers (Staples et al, 2008). Although - again as expected - tumour p53 mutation conferred an increased chance of relapse and death compared with having a WT p53 tumour (Olivier et al, 2006; Staples et al, 2008), it seems that deprivation is associated with a worse prognosis even amongst those with p53 mutation. Interestingly, for WT $p 53$ patients, this does not hold: all patients in deprivation category 10 with WT $p 53$ breast cancers remained disease free and survived. Reasons for this extreme difference are unclear; it is unlikely that patient selection (on the basis of the availability of tissue rather than consecutive patients by decile of residence) was responsible but suggests that the combination of $p 53$ mutation in breast cancer and extreme deprivation prejudices survival.

The influence of established prognostic features of breast cancer when comparing extreme deprivation (decile 10) patients with higher socio-economic status could not be excluded as contributors in the current series, with the biological features of poorer prognostic cancers (higher grade, ER negative and HER2 positive) in general more evident in the decile 10 patients. Moreover, the relationship between deprivation and ER positivity (Thomson et al, 2001) was not, as others have also found (Al Murri et al, 2004), confirmed in this study. The effects of treatment were also considered. The response of the p53 network in breast cancer to chemotherapies and radiation therapy may be dependent on the $p 53$ status of the cancer (Al Murri et al, 2004; Staples et al, 2008). The increased presence of $p 53$ mutations in the worst deprivation decile suggests such cancers may also be resistant to treatment and has implications for the types of chemotherapy that may be most effective (Staples et al, 2008).

From the results of multivariate analyses, patients from the worst deprivation group were significantly more likely to have a relapse and die from breast cancer, even after adjusting for other associated variables. Interestingly, p53 mutation status in itself was significantly associated with death when using Fisher's exact tests and KaplanMeier analyses, but not when analysed by binary logistic regression or Cox's regression. This suggests that $p 53$ mutation status has a strong interaction with (at least) one other variable in the model (i.e. a dominant 'partner'); therefore an interaction between $p 53$ mutation and deprivation decile 10 was sought. The $\beta$-coefficient of the interaction between $p 53$ mutation and deprivation category 10 was positive and statistically significant $(\beta=2.52, P<0.0001$ and $\beta=2.18$, $P<0.0001$ for death and recurrence, respectively). Moreover, the impact of the interaction was substantially greater than the additive effect (summation of the RR values) of the two variables alone, indicating a potentially synergistic relationship between deprivation and $p 53$ mutation both for survival and disease recurrence.

Among the pleiotropic roles of $p 53, p 53$ has a pivotal node in the inflammatory stress response pathway (Staib et al, 2005). In addition post-translational modification of $p 53$ by reactive nitrogen species (Hofseth et al, 2003) may lead to the selective clonal expansion of mutated p53 cells (Hofseth et al, 2003). As originally proposed by Virchow in the 19th century, links between inflammation and cancer have been established for both malignant and pre-malignant conditions (Hussain and Harris, 2007). However, although $p 53$ mutations have been identified in cancer-prone inflammatory diseases, including premalignant inflammatory 


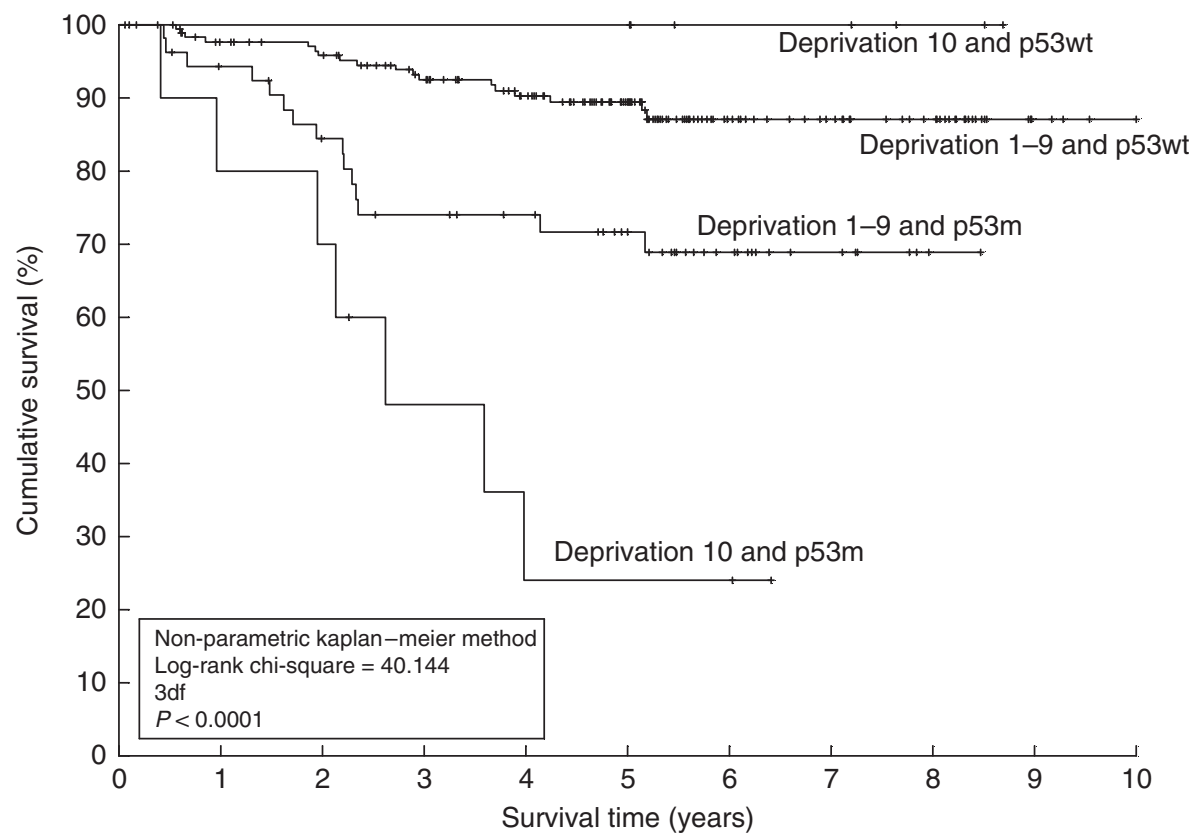

Number at risk

$\begin{array}{lccccccccccc}\text { Deprivation 1-9 and p53wt 174 } & 159 & 152 & 136 & 118 & 85 & 44 & 34 & 25 & 5 & 0 \\ \text { Deprivation 1-9 and p53m } & 54 & 48 & 41 & 35 & 32 & 26 & 15 & 7 & 1 & 0 & 0 \\ \text { Deprivation 10 and p53wt } & 7 & 7 & 7 & 7 & 7 & 7 & 4 & 4 & 2 & 0 & 0 \\ \text { Deprivation 10 and p53m } & 10 & 8 & 7 & 4 & 2 & 2 & 2 & 0 & 0 & 0 & 0\end{array}$

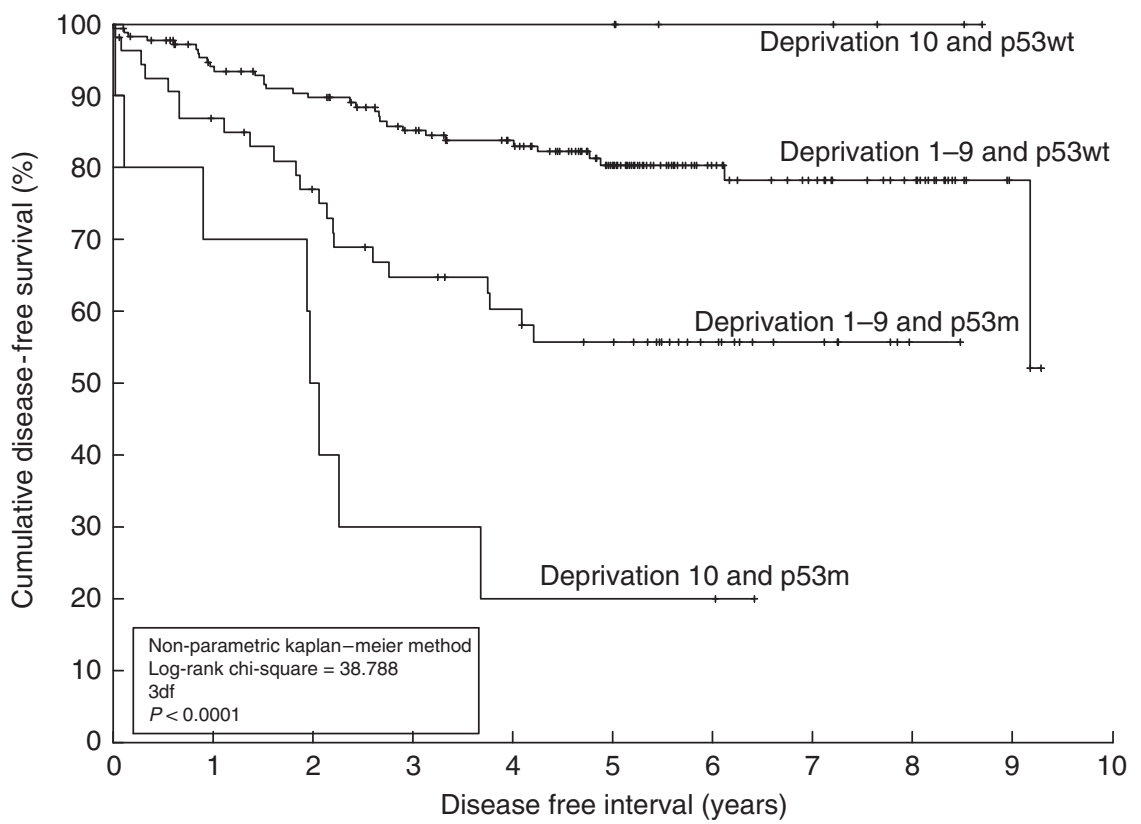

Number at risk

$\begin{array}{lccccccccccc}\text { Deprivation 1-9 and p53wt } & 175 & 154 & 144 & 127 & 113 & 79 & 40 & 30 & 21 & 3 & 0 \\ \text { Deprivation 1-9 and p53m } & 54 & 45 & 38 & 31 & 27 & 23 & 13 & 7 & 1 & 0 & 0 \\ \text { Deprivation 10 and p53wt } & 7 & 7 & 7 & 7 & 7 & 7 & 4 & 4 & 2 & 0 & 0 \\ \text { Deprivation 10 and p53m } & 10 & 7 & 5 & 3 & 2 & 2 & 2 & 0 & 0 & 0 & 0\end{array}$

Figure I Non-parametric Kaplan-Meier plots for overall survival (top) and disease-free survival (bottom) for four patient groupings - those in deprivation category 10 vs decile I -9 , and those having a p53 mutation vs wild-type p53. The logrank $\chi^{2}$ statistic (with associated degrees of freedom and $P$-value) was used to assess the statistical significance of difference in survival and disease-free chances. Patients with a p53 mutation had 5-year survivals of 72 and 24\% (deprivation groups I-9 and 10, respectively) and corresponding 5-year disease-free survivals of 56 and $20 \%$. Patients with wild-type p53 had 89 and $100 \%$ chance of surviving until 5 years (deprivation groups $1-9$ and 10, respectively), with corresponding 5 -year disease-free survivals of 80 and $100 \%$ 
conditions of the gastrointestinal tract (Hussain et al, 2000), to date no similar association has been proposed in breast cancer. Although the disparate range of $p 53$ mutations identified (data not shown) suggests a single agent is unlikely to be causative, patients in deprived communities do have a chronic inflammatory response that has been linked to breast cancer (Al Murri et al, 2004), colorectal cancer (McMillan et al, 2003) and increased coronary heart disease (Ridker et al, 2000; O'Reilly et al, 2006). Most recently, evidence that raised circulating markers of chronic inflammation (C-reactive proteins and serum amyloid A) have a threshold effect on breast cancer survival has emerged (Pierce et al, 2009). The tentative association explored between elevated C-reactive protein, serum amyloid A with ER- and progesterone receptor-negative cancers indirectly suggests further merit in pursuing a link between such inflammatory markers and $p 53$. We recognise that our findings need to be confirmed in another population of breast cancer patients, ideally, one with data on inflammatory markers and the standard breast cancer data.

p53 mutation, with potential aberrant inflammatory stress responses and therapeutic consequences, may account - at least in part - for the poor prognosis in women with breast cancer from the most deprived socio-economic background. This underlines the need to address the broader environment and social context of patient care along-side our increasing molecular understanding of cancer.

\section{ACKNOWLEDGEMENTS}

We thank the women who kindly donated breast tissue to the Tayside Tissue Bank for research purposes and all the clinicians, nurses, research nurses and administrative staff involved in this process. We also thank the Tayside Tissue Bank, the Health Division of the Scottish Government and C Heeley from Information Services Division, Scotland. We also thank L Kilburn from the Institute of Cancer Research Clinical Trials and Statistics Unit for review of the paper. This study was financially supported by Breast Cancer Research, Scotland.

Contributors: conception and design: LB, PRQ, AA, AMT; administrative support: AA, L-JB-S-B; provision of study materials or patients: J-CB, AMT, CAP, LBJ, NP, LW; collection and assembly of data: PRQ, AA, CMcC, L-JB-S-B; data analysis and interpretation: $\mathrm{LB}, \mathrm{AMT}, \mathrm{JAD}, \mathrm{CMCC}, \mathrm{PRQ}$; manuscript writing: LB, AMT, JAD; final approval of manuscript: LB, PRQ, NP, AA, LJB-S-B, CMcC, J-CB, CAP, LBJ, JAD, LW, AMT; LB had full access to all the data and had final responsibility for the decision to submit for publication.

\section{Conflict of interest}

NP and LW are employees of Roche Molecular Systems. All other authors declare no conflicts of interest.

\section{REFERENCES}

Al Murri AM, Doughty JC, Lannigan A, Wilson C, McArdle CS, McMillan DC (2004) The relationship between deprivation, tumour stage and the systemic inflammatory response in patients with primary operable breast cancer. Br J Cancer 91(6): 1063 - 1065

Banks J, Marmot M, Oldfield Z, Smith JP (2006) Disease and disadvantage in the United States and in England. JAMA 295: 2037-2045

Bishop J, Clark D, Harris V, Stockton D, Sutton M (2004) Deprivation and Urban Rural Measurement in ISD. Edinburgh. http://www.isdscotland. org/isd/files/Measuring-deprivation-in-ISD-v3-pdf

Bloom H, Richardson W (1957) Histological grading and prognosis in breast cancer; a study of 1409 cases of which 359 have been followed for 15 years. Br J Cancer 11(3): 359-377

Carstairs V, Morris R (1991) Deprivation and health in Scotland. University Press: Aberdeen

Detre S, Saclani Jotti G, Dowsett M (1995) A 'quickscore' method for immunohistochemical semiquantitation: validation for oestrogen receptor in breast carcinomas. J Clin Pathol 48(9): 876-878

Hofseth LJ, Saito S, Hussain SP, Espey MG, Miranda KM, Araki Y, Jhappan C, Higashimoto Y, He P, Linke SP, Quezado MM, Zurer I, Rotter V, Wink DA, Appella E, Harris CC (2003) Nitric oxide-induced cellular stress and p53 activation in chronic inflammation. Proc Natl Acad Sci USA 100: $143-148$

Hussain SP, Amstad P, Raja K, Ambs S, Nagashima M, Bennett WP, Shields PG, Ham AJ, Swenberg JA, Marrogi AJ, Harris CC (2000) Increased p53 mutation load in noncancerous colon tissue from ulcerative colitis: a cancer-prone chronic inflammatory disease. Cancer Res 60: 3333-3337

Hussain SP, Harris CC (2007) Inflammation and cancer: an ancient link with novel potentials. Int J Cancer 121(11): 2373-2380

Kelsey JL, Fischer DB, Holford TR, LiVoisi VA, Mostow ED, Goldenberg IS, White C (1981) Exogenous estrogens and other factors in the epidemiology of breast cancer. J Natl Cancer Inst 67(2): 327-333

Kogevinas M, Porta M (1997) Socioeconomic differences in cancer survival: a review of the evidence. IARC Sci Publ 138: 177-206

MacKie RM, Hole DJ (1996) Incidence and thickness of primary tumours and survival of patients with malignant melanoma in relation to socioeconomic status. Br Med J 312: 1125-1128

McMillan DC, Canna K, McArdle CS (2003) The effect of deprivation and the systemic inflammatory response on outcome following curative resection for colorectal cancer. Br J Cancer 89: 612-614

Mullee MA, De Stavola B, Romanengo M, Coleman MP (2004) Geographical variation in breast cancer survival rates for women diagnosed in England between 1992 and 1994. Br J Cancer 90(11): 2153-2156

Olivier M, Langerød A, Carrieri P, Bergh J, Klaar S, Eyfjord J, Theillet C, Rodriguez C, Lidereau R, Bièche I, Varley J, Bignon Y, Uhrhammer N, Winqvist R, Jukkola-Vuorinen A, Niederacher D, Kato S, Ishioka C, Hainaut P, Børreson-Dale A-L (2006) The clinical value of somatic TP53 gene mutations in 1794 patients with breast cancer. Clin Cancer Res 12(4): $1157-1167$

O'Reilly DStJ, Upton MN, Caslaske MJ, Robertson M, Norrie J, McConnachie A, Watt GCM, Packard CJ (2006) on behalf of the Midspan and WOSCOPS study groups. Plasma $\mathrm{C}$ reactive protein concentration indicates a direct relation between systemic inflammation and social deprivation. Heart 92(4): 533-535

Pierce BL, Ballard-Barbash R, Bernstein L, Baumgartner RN, Neuhouser ML, Wener MH, Baumgartner KB, Gilliland FD, Sorenson BE, McTiernan A, Ulrich CM (2009) Elevated biomarkers of inflammation are associated with reduced survival among breast cancer patients. J Clin Oncol 27(21): $3437-3444$

Quinn MJ, Cooper N, Rachet B, Mitry E, Coleman MP (2008) Survival from cancer of the breast in women in England and Wales up to 2001. Br J Cancer 99(Suppl 1): S53-S55

Ridker PM, Hennekens CH, Buring J, Rifai N (2000) C-reactive protein and other markers of inflammation in the prediction of cardiovascular disease in women. $N$ Engl J Med 342: 836-843

Sant M, Capocaccia R, Verdecchia A, Estève J, Gatta G, Micheli A, Coleman MP, Berrino F (1998) Survival of women with breast cancer in Europe: variation with age, year of diagnosis and country. The EUROCARE Working Group. Int J Cancer 77: 679-683

Schrijvers CT, Mackenbach JP, Lutz JM, Quinn MJ, Coleman MP (1995) Deprivation and survival from breast cancer. $\mathrm{Br} J$ Cancer 72: $738-743$

Schrijvers CT, Coebergh JW, Mackenbach JP (1997) Socioeconomic status and comorbidity among newly diagnosed cancer patients. Cancer 80: $1482-1488$

Staib F, Robles AI, Varticovski L, Wang XW, Zeeberg BR, Sirotin M, Zhurkin VB, Hofseth LJ, Hussain SP, Weinstein JN, Galle PR, Harris CC (2005) The p53 tumor suppressor network is a key responder to microenvironmental components of chronic inflammatory stress. Cancer Res 65: $10255-10264$

Staples O, Lain S, Ziyaie D, Thompson AM (2008) p53 and breast cancer. In: Walker RA, Thompson AM, editors Prognostic and predictive factors in breast cancer 2nd ed, pp 148-167. Informa Healthcare: London, UK

Taylor A, Cheng KK (2003) Social deprivation and breast cancer. J Public Health Med 25: 228-233 
Thomson CS, Hole DJ, Twelves C, Brewster DH, Black RJ (2001) Prognostic factors in women with breast cancer: distribution by socio-economic status and effect on differences in survival. J Epidemiol Community Health 55: $308-315$

Thomson CS, Brewster DH, Dewar JA, Twelves CJ (2004) on behalf of the Scottish Cancer Therapy Network. Improvements in survival for women with breast cancer in Scotland between 1987 and 1993: impact

of earlier diagnosis and changes in treatment. Eur J Cancer 40(5): $743-753$

Twelves CJ, Thomson CS, Dewar JA, Brewster DH (2001) Variation in survival of women with breast cancer: Health Board remains a factor at ten years. Br J Cancer 85: 637-640

Vousden KH, Lane DP (2007) p53 in health and disease. Nat Rev Mol Cell Biol 8: $275-283$ 